MRS Advances (C) 2016 Materials Research Society

DOI: 10.1557/adv.2016.106

\title{
Graphene Channels Interfaced with Quantum Dots in Field Effect Transistors: Electrical and Photo-Induced Effects
}

\author{
Xin Miao ${ }^{1}$, Samarth Trivedi ${ }^{2}$, and Haim Grebel ${ }^{1}$ \\ ${ }^{1}$ Electronic Imaging Center and ECE Dept., New Jersey Institute of technology (NJIT), \\ Newark, NJ 07102, U.S.A \\ ${ }^{2}$ Department of Chemistry, New Jersey Institute of technology (NJIT), \\ Newark, NJ 07102, U.S.A grebel@njit.edu
}

\begin{abstract}
Graphene-based field effect transistors (GFETs) were assessed when interfaced with well separated and precisely placed core/shell CdSe/ZnS semiconductor quantum dot (QD) arrays. The QDs were imbedded in a hexagonal hole-array, which was formed in a layer of anodized aluminum oxide on $\mathrm{Si} / \mathrm{SiO}_{2}$ substrates. Graphene (single, or two layers), grown by chemical vapor deposition (CVD) on $\mathrm{Cu}$ foils, was transferred and placed on top of the QDs imbedded films and served as the transistor channel. Electrical characteristics under white-light illumination at various biasing conditions revealed that the photo current was decreasing upon increasing biasing. The device's photoluminescence (PL) as a function of both the drain-source and gate-source potentials also reduced as a function of the potential biases. We observed two maxima in the PL data while tilting the sample with respect to the incident laser beam. We attributed it to the optimal coupling between the incident and the emission wavelengths to resonating surface modes.
\end{abstract}

\section{INTRODUCTION}

Graphene - a two-dimensional atomic layer of graphite - has outstanding physical properties: high conductivity, chemical inertness, mechanical robustness and unusual electronic dispersion relations [1]. The electrical properties of free-standing graphene layer(s) have been studied in the past when deposited over nano-pore arrays, which were formed in anodized aluminum oxide (AAO) [2-3]. Raman spectra of the graphene lines were accentuated by the hole-array, as well. The periodic array of nano-pores has led to the realization of the first visible surface plasmon laser [4-6]; there, one takes advantage of simultaneous resonating plasmon/polariton modes in both the pump and the emission frequencies. Here, we took advantage of surface modes, which were propagating along the graphene channels. We analyzed the effect of electrical bias on the $\mathrm{PL}$ of well-separated and pore imbedded core/shell CdSe/ZnS semiconductor QDs when interfaced with one or two-layer graphene channels. The interplay between the electrical and optical bias of the channel may lead to new opto-electronic elements.

\section{EXPERIMENT}

Depicted in Figure 1a, we started with $\mathrm{Si} / \mathrm{SiO}_{2}$ substrates. The $<100>$ p-type $\mathrm{Si}$ wafer was serving as the back-gate electrode. The silicon substrates were deposited with $150 \mathrm{~nm}$ of $\mathrm{SiO}_{2}$ using plasma assisted CVD. A $250 \mathrm{~nm}$ thick layer of aluminum was evaporated on top of the 
$\mathrm{SiO}_{2}$ substrates. The aluminum was later anodized completely by a two-step anodization process [6-7]. As a result of the anodization of aluminum, a 50-100 nm thick perforated layer of alumina was formed on top of the $\mathrm{Si} / \mathrm{SiO}_{2}$. The diameter of the holes was ranging between 25 and $30 \mathrm{~nm}$. The pitch of the hexagonal hole-array was ca $100 \mathrm{~nm}$. Core/shell semiconductor CdSe/ZnS QDs with an average diameter of $\sim 4 \mathrm{~nm}$ (Ocean NanoTech, AR) and coated with octadecylamine (for better suspension and agglomeration prevention) were first suspended in toluene and drop-casted on the AAO layer. Some of the QDs were imbedded in the pores while others remained on the surface and were wiped out. The graphene was grown on $\mathrm{Cu}$ foils using methane gas [8] and were deposited later with a $250 \mathrm{~nm}$ layer of PMMA. The $\mathrm{Cu}$ foil was completely etched away using ferric acid and the suspended films were washed successively with water until clear. Then, the film was transferred on top of the QD imbedded AAO. The films' area was large, approximately $1 \mathrm{~cm}^{2}$. It is our experience that film contamination would result in a very large channel impedance and low carrier mobility [9] which is not so apparent in our data. Nevertheless, recent studies show that the $\mathrm{Cu}$ and $\mathrm{Fe}$ contamination of graphene may well exceed $10^{13}$ atoms $/ \mathrm{cm}^{2}$ during the growth and etch stages [10]. Assuming a uniform contamination level of $10^{14}$ atoms $/ \mathrm{cm}^{2}$ and a spherical QD in contact with the graphene layer, this means that only $\sim 1$ contaminant atom is interfacing with the QD. Raman spectroscopy ascertained that the graphene was consisted of at most two layers. The current-voltage curve between the source and drain $\left(I_{d s}-V_{d s}\right.$ curve $)$ was linear in the absence of light or the absence of gate bias (Figure 1b) alluding to a good ohmic contact.

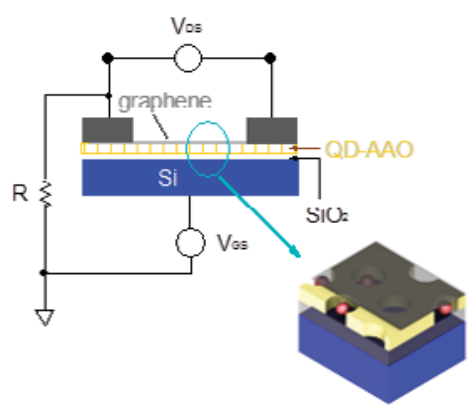

(a)

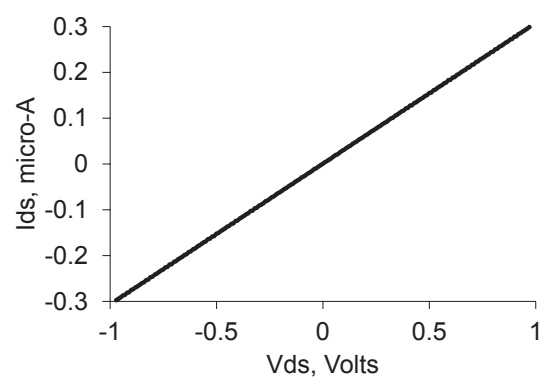

(b)

Figure 1. (a) The GFET configuration. (b) $I_{d s}-V_{d s}$ curve for $V_{g s}=0$ and under dark conditions.

Current-Voltage curves ( $I-V$ curves) were taken with a probe station at a resolution of 100 fA. The samples were illuminated by a white light tungsten source at various intensities. The fiber interfaced source was focused and placed approximately $20 \mathrm{~cm}$ from the samples. The source had two arms: one was illuminating a reference power meter while the other arm was illuminating the sample. The PL data were obtained with a $35 \mathrm{~mW}$ Ar ion laser at $488 \mathrm{~nm}$ in a confocal arrangement and using a $20 \mathrm{~cm}$ monochromator, a PMT, an optical chopper and a lockin amplifier. The laser spot illuminated the entire area between the source and drain electrodes $\left(\sim 0.5 \mathrm{~cm}^{2}\right)$. The sample was placed on a rotatable platform to enable coupling to surface modes (see below). 


\section{RESULTS AND DISCUSSION}

Electrical characteristics of the GFETs under white-light illumination were studied. We compared the channel current under illumination and under dark conditions. This method is very effective in revealing small conductance changes. The channel current was plotted as a function of $V_{d s}$ and $V_{g s}$ for a fixed intensity value of the white-light intensity. In Figure 2, we show such a map of the differential current $\left[\left(I_{d s}\right.\right.$ under white light $)-\left(I_{d s}\right.$ in dark $\left.)\right]$ plotted as a function of the drain-source, $V_{d s}$ and the gate-source, $V_{g s}$ voltages.

As can be seen from Figure 2a, the differential current $\Delta I_{d s}$ decreased as a function of $V_{g s}$. Similarly, varying $V_{d s}$ only slightly decreased the differential current, $\Delta I_{d s}$. The map is symmetrical with respect to $V_{d s}$ as may be expected for a linear system. When the sample was exposed to a larger intensity of the white light source (Figure $2 b$ ), the surface plot became more regular. One can explain these trends as follows: there are two competing effects when the sample is illuminated by a white-light source. On one hand, the QDs are contributing to the channel photo-current whereas when ionized, they reduce the overall channel current. The QD are of p-type and the biasing affects their energy band diagram.

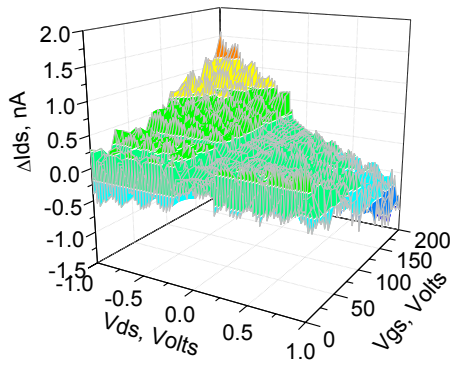

(a)

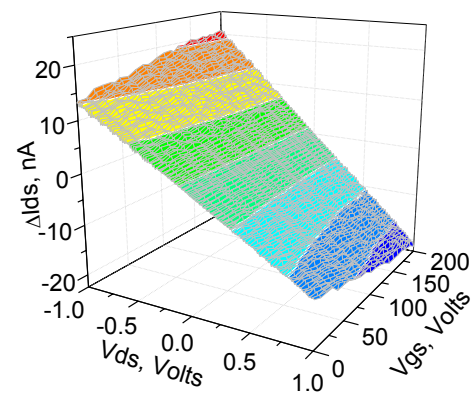

(b)

Figure 2. The difference in $I_{d s}$ between illuminated and non-illuminated cases as a function of $V_{d s}$ and $V_{g s}$. The white light intensity was (a) $320 \mathrm{~mW} / \mathrm{cm}^{2}$ and (b) $500 \mathrm{~mW} / \mathrm{cm}^{2}$.

A plot of the channel's photo-current (as opposed to the differential current) as a function of white light intensity is shown in Figure 3. Maximum photo-current is observed for $380 \mathrm{~mW} / \mathrm{cm}^{2}$. Clearly, the channel conductance is substantially suppressed under strong light illumination. Typically, the absorption of graphene is constant of wavelength, approximately $2.3 \%$ per layer. For graphene only films one expects that the photo-current would increase as a function of increasing light intensity. Therefore, any decrease in the photo-current as a function of light intensity, ought to be attributed to ionization of the QDs and the increase in the channel impedance. Contamination of the channels (if any) by $\mathrm{Cu}$ or Fe atoms is not expected to contribute to the differential photo-current. 


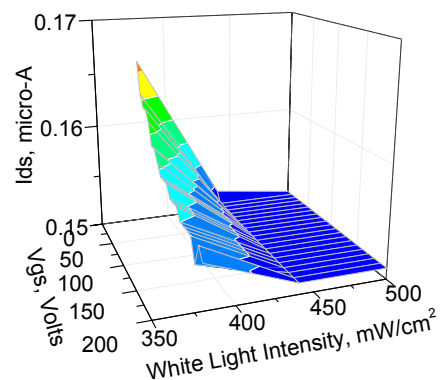

Figure 3. $I_{d s}$ as a function of $V_{g s}$ and white light intensity at $V_{d s}=0.5 \mathrm{~V}$. Clearly, the channel current is decreasing as a function of either the light intensity or the gate voltage.

Photoluminescence of the QDs as a function of $V_{d s}$ and $V_{g s}$ was also assessed. The peak luminescence of QDs was at $590 \mathrm{~nm}$ (Figure $4 \mathrm{~b}$ ). The PL change was more pronounced when measured as a function of $V_{g s}$, again alluding to the shift in the energy levels of the p-type QDs by the gate bias. Figure 4a indicates that the PL peak decreased monotonically as a function of $V_{g s}$. A slight decrease was also noted as a function of $V_{d s}$ (Figure 5).

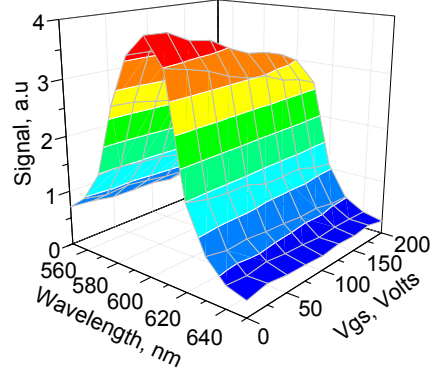

(a)

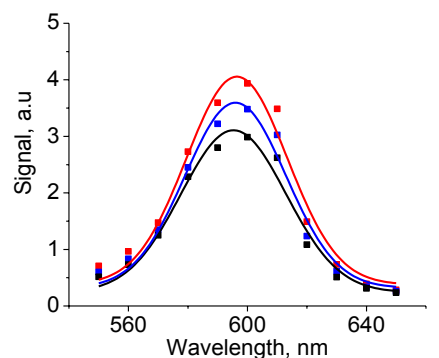

(b)

Figure 4. (a) PL of QDs as a function of $V_{g s}$ at normal incidence and at $V_{d s}=0.1 \mathrm{~V}$.

(b) PL as a function of wavelength at $V_{g s}=0$ (top, red curve), 100 (middle, blue curve), $200 \mathrm{~V}$ (bottom, black curve), respectively. The source-drain voltage was, $V_{d s}=0.1 \mathrm{~V}$ and the intensity of the $488 \mathrm{~nm}$ laser was $35 \mathrm{~mW}$, illuminating the entire area between the electrodes $\left(\sim 0.5 \mathrm{~cm}^{2}\right)$. There is a small but noticeable PL peak shift towards the shorter wavelengths. 


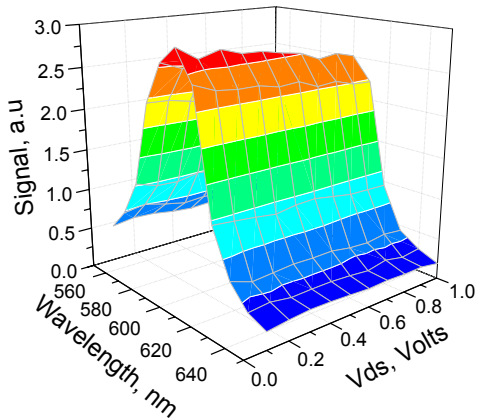

Figure 5. PL as a function of $V_{d s}$ at normal incidence at $V_{g s}=50 \mathrm{~V}$.

Coupling to surface modes: The QDs were imbedded in a thin periodic and perforated structure, which served as a surface guide. The electromagnetic surface mode was bound on one side by the low index of $\mathrm{SiO}_{2}$ layer $\left(n_{\mathrm{SiO} 2} \sim 1.5\right)$ and the graphene/air from the other side $\left(n_{\text {air }}=1\right.$; an approximation for graphene may be taken as, $n_{\text {graphene }} 2.6-1.3 \mathrm{i}$ [11]). The electromagnetic radiation may be efficiently coupled into a surface mode when the wave-vector of either the incident, or the scattered (or both) waves are at resonance with the wave-vector of the perforated substrate. At resonance the surface mode becomes a standing wave. Since the QDs are embedded in a well-defined hole-array, their positions are in-phase with the standing electromagnetic surface mode. In that case, the PL signal would be enhanced due to optimal coupling between the QD and the surface mode. As one tilts the sample with respect to the incident laser beam, a series of PL maxima and minima are to be expected as detailed below.

The optimal launching conditions for a surface mode is achieved by a small tilt and in-plane rotation of the perforated substrate with respect to the p-polarized incident beam (The incident beam was polarized such that it had a polarization component perpendicularly to the sample's surface). Note that the array pitch is much smaller than the propagating wavelength and a bound surface mode is utilizing every other or even larger number of hole-planes. The tilt angle $\theta$ may be computed similarly to [12] as,

$$
\sin (\theta)=\frac{\lambda_{0}}{a} \sqrt{\left(\frac{4}{3}\right)\left(q_{1}^{2}-q_{1} q_{2}+q_{2}^{2}\right)}-n_{\text {eff }}
$$

Here, $\lambda_{0}$ is the incident or emitted wavelength, $a$ is the pitch for the holes array ( $a \sim 100 \mathrm{~nm}$ ), $q_{1}$ and $q_{2}$ are sub-integers (e.g., 1/5) representing the ratio between the array pitch and the propagating wavelength. The effective refractive index for the graphene may be estimated using the equation for surface waveguides, which are bound by alumina [13]: using the real and imaginary constants of the refractive index for graphene, we find that the propagation constant is $\sim 12 \mu \mathrm{m}^{-1}$ for $\lambda=0.6 \mu \mathrm{m}$. The attenuation coefficient is $\sim 0.9 \mu \mathrm{m}^{-1}$. This is translated to an effective index $n_{\text {eff }} \sim 1.2$ in Eq. 1, while the attenuation coefficient limits the effective propagation distance to $1.1 \mu \mathrm{m}$ (or $\sim 11$ pitch lengths).

In the experiments, the GFETs sample was first rotated in-plane to achieve maximum signal and then tilted with respect to the incident wave-vector. As observed from Figure 6, there are 
two PL signal peaks as a function of the tilt angle: one peak is situated at $\theta=0^{\circ}$ and the other, as we approach $\theta \sim 7^{\circ}$ (the maximum tilt angle available in our system). The first PL maxima may be attributed to the resonating surface mode at the excitation wavelength $(\lambda=488 \mathrm{~nm})$ with $q_{1}=1 / 5$ and $q_{2}=0$. The other peak may be attributed to the resonating surface mode at the emission wavelength $\left(\lambda=590 \mathrm{~nm}\right.$ ) with $q_{1}=1 / 5$ and $q_{2}=0$. Using Eq. 1 for $\lambda=590 \mathrm{~nm}$, we obtain $\theta \sim 9^{\circ}-$ in reasonable agreement with the experimental data. As a reference experiment, we measured QDs on a flat glass slide. The PL signal decreased monotonically as a function of the tilt angle as expected: as the flat substrate is tilted, the illuminated area is increased and the intensity per area is decreased as $\cos (\theta)$, leading to reduction in the overall PL signal.

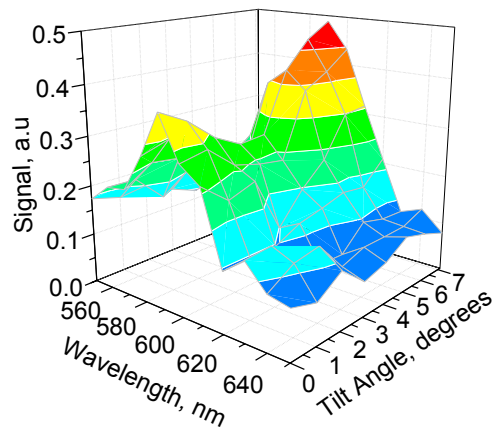

Figure 6. Photoluminescence as a function of the tilt angle.

\section{CONCLUSIONS}

In summary, the electrical and optical properties of graphene channels were studied when interfaced with well-separated and periodically placed quantum dots. Photoluminescence has peaked when either the incident or the emission wavelengths were effectively coupled to surface modes.

\section{REFERENCES}

1. K.S. Novoselov, A.K. Geim, S.V. Morozov, D. Jiang, Y. Zhang, S.V. Dubonos, I.V. Grigorieva and A.A. Firsov, "Electric Field Effect in Atomically Thin Carbon Films", Science, 306, 666 (2004).

2. A. Banerjee, R. Li and H. Grebel, "Surface Enhanced Raman with Graphenated Anodized Aluminum Oxide Substrates: the Effect of Sub-Wavelength Patterns", Nanotechnology, 20 (29), art. no. 295502 (2009).

3. A. Banerjee and H. Grebel, "Depositing Graphene Films on Solid and Perforated Substrates", Nanotechnology, 19 1-5 art. no. 365303 (2008).

4. Ruiqiong Li, and Grebel Haim, "Surface Enhanced Fluorescence", IEEE Sensors, 10(3), 465468 (2010) doi: 10.1109/JSEN.2009.2038513.

5. R. Li, A. Banerjee and H. Grebel, "The possibility for surface plasmon lasers", Optics Express, 17, 1622-1627 (2009). 
6. A. Banerjee, R. Li and H. Grebel, "Surface plasmon lasers with quantum dots as gain media", Appl. Phys. Letts., 95, 251106 (2009); doi:10.1063/1.3276273.

7. A. Banerjee, R-Q. Li and H. Grebel, "Raman Spectrum of Graphene Coated Nano-Holes", Mater. Res. Soc. Symp. Proc. Vol. 1059, 2008 Materials Research Society 1059-KK10-26.

8. Xuesong Li, Yanwu Zhu, Weiwei Cai, Mark Borysiak, Boyang Han, David Chen, Richard D. Piner, Luigi Colombo and Rodney S. Ruoff, "Transfer of Large-Area Graphene Films for High-Performance Transparent Conductive Electrodes”, Nano Letts, 9(12), 4359-4363 (2009).

9. Amrita Banerjee and Haim Grebel, "Freestanding graphene and its applications", ECS Transactions Vol 19(5), 2009.

10. Grzegorz Lupina et al, "Residual Metallic Contamination of Transferred Chemical Vapor Deposited Graphene", ACS Nano, 2015, 9, 4776-4785.

11. P. Blake, E. W. Hill A. H. Castro Neto K. S. Novoselov, D. Jiang, R. Yang, T. J. Booth, A. K. Geim, "Making graphene visible", arXiv:0705.0259v3 [cond-mat.mes-hall] 22 Sep 2007.

12. H. Grebel, "Linear and non-linear phenomena with resonating Surface Polariton waves and their applications", in Structured Surfaces as Optical Metamaterials, Alexei A. Maradudin. Editor, Cambridge, 2011

13. A. Yariv and P-C Yeh, Optical waves in Crystals, Wiley and Sons, 1984, Ch 11. 